\title{
Novel PKD1 and PKD2 mutations in Taiwanese patients with autosomal dominant polycystic kidney disease
}

\author{
Ming-Yang Chang ${ }^{1}$, Hsiao-Mang Chen ${ }^{2}$, Chang-Chyi Jenq ${ }^{1}$, Shen-Yang Lee ${ }^{1}$, Yu-Ming Chen ${ }^{1}$, Ya-Chung Tian ${ }^{1}$, \\ Yung-Chang Chen ${ }^{1}$, Cheng-Chieh Hung ${ }^{1}$, Ji-Tseng Fang ${ }^{1}$, Chih-Wei Yang ${ }^{1}$ and Yah-Huei Wu-Chou ${ }^{2}$
}

Autosomal dominant polycystic kidney disease (ADPKD) is a heterogeneous disease caused by mutations in PKD1 and PKD2. The genotype-phenotype correlations are not completely understood. We performed direct PCR-sequencing plus multiplex ligation-dependent probe amplification for PKD1 and PKD2 in 46 unrelated patients. Disease-causing mutations were identified in $30(65 \%)$ patients: 23 (77\%) patients have mutations in PKD1 and 7 (23\%) have mutations in PKD2. Nonsense, splicing or frame-shifting mutations were found in 18 patients, exon duplication in 1 and missense mutations in 11 patients. Two likely PKD1 hypomorphic alleles (p.Arg2477His and p.Arg3439Trp) segregated with mild disease in a family. A total of 34 mutations were identified and $17(50 \%)$ of which are novel. The median age at onset of dialysis was significantly earlier in patients with PKD1 mutations (52 years) than in patients with PKD2 mutations (65.5 years) and those with an undetermined genotype (67 years) by survival analysis (log-rank test, $P=0.014$ ). Patients carrying $P K D 1$-truncating mutations have a trend toward earlier initiation of dialysis compared with carriers of non-truncating mutations (52 years vs 57 years, $P=0.061)$. A family history of dialysis before 55 years was more common in PKD1 patients than in others $(P<0.05)$. In conclusion, this study identified novel mutations in PKD1 and PKD2 and demonstrated the presence of PKD1 hypomorphic alleles in Taiwanese patients. Patients carrying PKD1 mutations, especially those with truncating mutations, could have a more rapidly progressive disease than others. These results might have implications for diagnosis and risk stratification in patients with ADPKD. Journal of Human Genetics (2013) 58, 720-727; doi:10.1038/jhg.2013.91; published online 29 August 2013

Keywords: mutation; PKD1; PKD2; polycystic kidney disease

\section{INTRODUCTION}

Autosomal dominant polycystic kidney disease (ADPKD) is a common inherited disorder resulting from mutations in PKD1 (MIM\#601313) and PKD2 (MIM\#173910) genes. $^{1}$ In clinically defined populations, mutations in PKD1 and PKD2 account for $85 \%$ and $15 \%$ of cases, respectively. ${ }^{2}$ This slowly progressive disease is not only associated with multiple kidney cysts but also causes systemic manifestations, such as liver cysts and cerebral aneurysms. ${ }^{3}$ The protein products of PKD1 and PKD2 are polycystin-1 (PC-1) and PC-2, respectively. These two proteins are critical for maintaining normal renal tubular structure during kidney development. ${ }^{4-6}$ PC-1 and PC-2 have been found in primary cilia to act as a mechanosensor ${ }^{7,8}$ and between cells or at cell-matrix attachments to mediate cell adhesion.9,10 End-stage renal disease (ESRD) is expected in nearly $50 \%$ of ADPKD patients at the age of 60 years. ${ }^{11}$
Patients with PKD1 mutations usually have an average age at onset of ESRD earlier than patients with PKD2 mutations (53.4 years vs 72.7 years). ${ }^{12,13}$ Both locus and allelic heterogeneity in the $P K D$ genes may influence the rate of disease progression, ${ }^{14,15}$ but the genotype/ phenotype correlations are not entirely clear.

Molecular diagnostics for ADPKD has become available and increasingly important in recent years. ${ }^{2}$ Traditional diagnostic approach is based on renal ultrasound that cannot exclude the disease in at-risk individuals until the age of 40 years, especially in families with mutations in PKD2. ${ }^{16}$ PCR and direct sequencing analysis can provide direct evidence of $P K D$ mutations, while multiplex ligation-dependent probe amplification (MLPA) may detect large deletion/duplication mutations that cannot be detected by direct sequencing. ${ }^{17,18}$ However, there are no mutation hot spots in PKD genes. ${ }^{2}$ Furthermore, interpretation of the identified missense

${ }^{1}$ Kidney Research Center and Department of Nephrology, Chang Gung Memorial Hospital, Chang Gung University College of Medicine, Taoyuan, Taiwan and ${ }^{2}$ Human Molecular Genetics Laboratory, Chang Gung Memorial Hospital, Chang Gung University College of Medicine, Taoyuan, Taiwan

Correspondence: Dr M-Y Chang, Kidney Research Center and Department of Nephrology, Chang Gung Memorial Hospital, Chang Gung University College of Medicine, 5 Fu-Shing Street, Kueishan, Taoyuan 333, Taiwan.

E-mail: mingyc@adm.cgmh.org.tw

or Dr Y-H Wu-Chou, Human Molecular Genetics Laboratory, Chang Gung Memorial Hospital, Chang Gung University College of Medicine, 5 Fu-Shing Street, Kueishan, Taoyuan 333, Taiwan.

E-mail: yhwc6333@adm.cgmh.org.tw

The results of this study were presented as a poster at the Annual meeting of the American Society of Nephrology, 1-4 November 2012, San Diego, CA, USA.

Received 11 June 2013; revised 19 July 2013; accepted 2 August 2013; published online 29 August 2013 
mutations can be challenging, because multiple sequence variants exist in PKD1 and PKD2. ${ }^{19}$ Recent studies have demonstrated the existence of incompletely penetrant $P K D 1$ mutations. ${ }^{20-22}$ The combination of these hypomorphic PKD1 alleles may cause typicalto-severe cystic disease, whereas one such allele may cause unusually mild disease. ${ }^{23}$ The Polycystic Kidney Mutation Database (PKDB; http://pkdb.mayo.edu) is a continually updated online reference describing all published $P K D$ mutations and variants. ${ }^{24,25}$ A collection of more mutation data of $P K D$ genes from different populations would allow better interpretation of the results of genetic testing.

Although PKD mutations from different countries have been reported, data in the Taiwanese population are still lacking. ${ }^{19,26}$ Here we report on our single-center experience of direct sequencing and MLPA for PKD1 and PKD2 in 46 unrelated Taiwanese patients with an ultrasound diagnosis of ADPKD. The specific purpose was to examine the characteristics of mutations in our cohort and their correlations to disease progression.

\section{PATIENTS AND METHODS}

\section{Patients}

A total of 46 unrelated patients with a clinical diagnosis of ADPKD at the Chang Gung Memorial Hospital, Linkou, Taiwan were enrolled between October 2008 and May 2011. All participants signed written informed consent. Approval for this work had been obtained from the Institutional Review Board Committee at the Chang Gung Memorial Hospital. The study was conducted in adherence to the Declaration of Helsinki. The diagnosis of ADPKD was made according to the renal ultrasound criteria. ${ }^{27}$ In the absence of a family history of ADPKD, bilateral renal enlargement and at least 10 cysts in each kidney were required for diagnosis. ${ }^{28}$ Blood samples were obtained from these patients and their available families members. Control DNA was obtained from a database of healthy volunteers.

\section{Clinical assessment}

Patients were interviewed for family and medical histories. A family history of early dialysis (before the age of 55 years) and late dialysis (after the age of 65 years or no need for dialysis) due to ADPKD was recorded. ${ }^{13}$ Clinical and laboratory data were obtained from medical records. An estimated glomerular filtration rate (eGFR) was calculated according to the Modification of Diet in Renal Disease study equation. ${ }^{29}$

\section{Mutation analysis of PKD1 and PKD2}

Genomic DNA was extracted from lymphocytes harvested from venous blood samples. Direct sequencing of exonic and flanking introgenic regions of all PKD1 and PKD2 exons was performed as previously published methods. ${ }^{25,30}$ For amplifying the duplicated part of PKD1 (exons 1-34), long-range PCR was first performed. Seven distinct long-range PCR fragments (exon 1, exons 2-7, exons $8-12$, exons $13-15$, exons $15-21$, exons $22-28$ and exons 29-34) were amplified using PKD1-specific primers. ${ }^{25,30}$ Exons $35-46$ of PKD1 and exons 1-15 of PKD2 were amplified from genomic DNA according to previously described PCR primers and conditions. ${ }^{30,31}$ The PCR products were purified and sequenced on an ABI3730 autosequencer (Applied Biosystems, Foster City, CA, USA). The pathogenicity of missense variants were evaluated using SIFT, PolyPhen and GVGD prediction programs as described previously. ${ }^{20}$ The 1000 Genomes Project data (http://browser.1000genomes.org), the Exome Sequencing Project (http://evs.gs.washington.edu/EVS) and the SingleNucleotide Polymorphism Database were checked for the reported sequence variants. The Human Splicing Finder software (http://www.umd.be/HSF/) was used to predict splicing signal. ${ }^{32}$ PKDB (http://pkdb.mayo.edu) was consulted for the previously reported mutations. The recommendation of HGVS (Human Genome Variation Society) for mutation nomenclature was followed. Nucleotide changes were numbered according to the coding sequences of PKD1 (NM_001009944.2) and PKD2 (NM_000297.3).

\section{MLPA}

Screening for $P K D 1 / P K D 2$ exon deletion/duplication was performed using MLPA according to the manufacturer's instructions. The MLPA kit (SALSA MLPA KIT P351-B1/P352-B1 PKD1-PKD2) was purchased from MRC-Holland (Amsterdam, The Netherlands).$^{33}$ It contains $P K D 1$ probes for 37 of the 46 exons and 15 PKD2 probes covering all PKD2 exons. Data were analyzed using the Coffalyser MLPA analysis tool (MRC-Holland, Amsterdam, The Netherlands).

\section{Real time quantitative PCR (Q-PCR)}

MLPA revealed one family with significant copy number variation of probe for PKD1 exon 1. To validate the results of MLPA, a real-time Q-PCR was performed using Custom Taqman Copy Number Assays (Applied Biosystems, Carlsbad, CA, USA). A set of commercially designed primers against the binding site of MLPA PKD1 exon 1 probe was used (Assay ID: PKD1E1_CCS07DN). The reaction was performed in triplicate for each sample.

\section{Statistical analysis}

Continuous variables are expressed as mean \pm s.d. and compared using oneway analysis of variance. Categorical variables were analyzed using the Fisher's exact or Chi-square test. Cumulative renal survival curves were generated by the Kaplan-Meier method and compared with the log-rank test. $P$-values $<0.05$ were considered statistically significant. All analyses were performed using the Graphpad Prism 5.1 (Graphpad, La Jolla, CA, USA).

\section{RESULTS}

\section{Clinical characteristics and genotyping}

We performed a complete mutational analysis using direct sequencing and MLPA analysis of PKD1 and PKD2 in 46 unrelated patients with the diagnosis of ADPKD by ultrasound. We found disease-causing mutations in $30(65 \%)$ patients: $23(77 \%)$ patients have PKD1 mutations and 7 (23\%) have PKD2 mutations. The remaining 16 (35\%) were categorized as undetermined genotype either because no mutations were detected or the identified variants were interpreted as nonpathogenic variants.

Table 1 summarizes the clinical characteristics in the study cohort according to their genotypes. There were 24 male and 22 female patients. The mean age was $47.5 \pm 13.0$ years. ADPKD family history was reported in $35(76 \%)$ patients. Family history of ADPKD with early dialysis (age $\leqslant 55$ years) was found in 11 (24\%) patients, whereas late dialysis (age $>65$ years) was reported in $8(17 \%)$ patients. Hypertension was noted in $31(67.4 \%)$ patients while gross hematuria, urinary tract infection and urolithiasis were found in 13 (28.3\%), $16(34.8 \%)$ and $19(41.3 \%)$ patients, respectively. Subarachnoid hemorrhage was noted in $1(2.2 \%)$ patient and two patients reported documented cerebral aneurysms. Mitral valve prolapse was noted in $1(2.2 \%)$ patient. Extrarenal cysts in the liver, pancreas and spleen were detected in $29(63 \%)$ patients. In patients with PKD1 mutations, the ages at recruitment tended to be younger than the other two groups $(P=0.09)$. A family history of dialysis after 65 years was more frequent in patients with $P K D 2$ mutations, whereas a family history of dialysis before 55 years was more frequent in patients with PKD1 mutations $(P<0.05)$. Other clinical parameters and antihypertension medication use were not significantly different between genotypes.

The kidney sizes as estimated by ultrasound were not different between genotypes. However, patients with PKD1 mutations in our study tended to be younger than patients with PKD2 mutations, suggesting that kidney enlargement may occur earlier in PKD1 patients than in PKD2 patients. 
Renal survival analysis

We then performed a Kaplan-Meier survival curve analysis to compare the age at onset of ESRD between different genotypes. As shown in Figure 1a, PKD1 patients $(n=23)$ have a significantly lower renal survival curve than $\operatorname{PKD} 2$ patients $(n=7)$ and patients with an undetermined genotype $(n=16)$ (log-rank test, $P=0.014)$. The median age of ESRD was 52 years in patients with PKD1 mutations ( $n=7$, range $40-58$ years), 65.5 years in patients with $P K D 2$ mutations $(n=2$, range 64-67 years) and 67 years in patients with an undetermined genotype ( $n=6$, range, $41-79$ years). We then investigated whether the type of PKD1 mutation influenced the age at onset of ESRD. We found a trend of earlier initiation of dialysis in patients with $P K D 1$-truncating mutations $(n=14)$ compared with patients with PKD1 missense mutations $(n=9)$ as shown in Figure $1 \mathrm{~b}$ (log-rank test, $P=0.061$ ). The median age of ESRD in patients with and without $P K D 1$-truncating mutations was $52(n=5)$ and 57 $(n=2)$ years, respectively.

\section{Characteristics of mutations}

Using direct PCR sequencing, we found 15 definite pathogenic mutations (12 in PKD1 and 3 in PKD2) in 17 (35\%) probands (Tables 2 and 3). Among these mutations, 9 (60\%) were nonsense mutations, $5(33 \%)$ were frame-shifting and $1(7 \%)$ was splicing mutation. We identified two novel PKD2 missense mutations (p.Try392Asp and p.Ala389Val) predicted to be likely pathogenic (Table 3). Fifteen PKD1 missense mutations, which were predicted to be likely pathogenic, were identified in nine patients (Table 4). A deletion mutation in PKD1 intron 38 (c.IVS38-100_98del3) predicted to generate new splice sites was found in one patient who started maintenance dialysis at the age of 40 years (Table 4 ). One patient (CG44) was found to have a PKD1-truncating mutation (p.Glu2810X) and four likely pathogenic missense mutations (Table 4). She experienced right middle cerebral artery infarction at the age of 45 years and an aneurysm was found in the right cranial internal carotid artery by angiography. She had advanced chronic kidney disease (eGFR $15 \mathrm{ml}$ per min per $1.73 \mathrm{~m}^{2}$ ) at the age of 50 years. In total, we identified 119 different sequence variants of PKD1 and PKD2: 15 (13\%) are definite pathogenic mutations, $17(14 \%)$ are likely pathogenic mutation, $50(42 \%)$ are likely non-pathogenic missense mutation and $37(31 \%)$ are silent polymorphism.
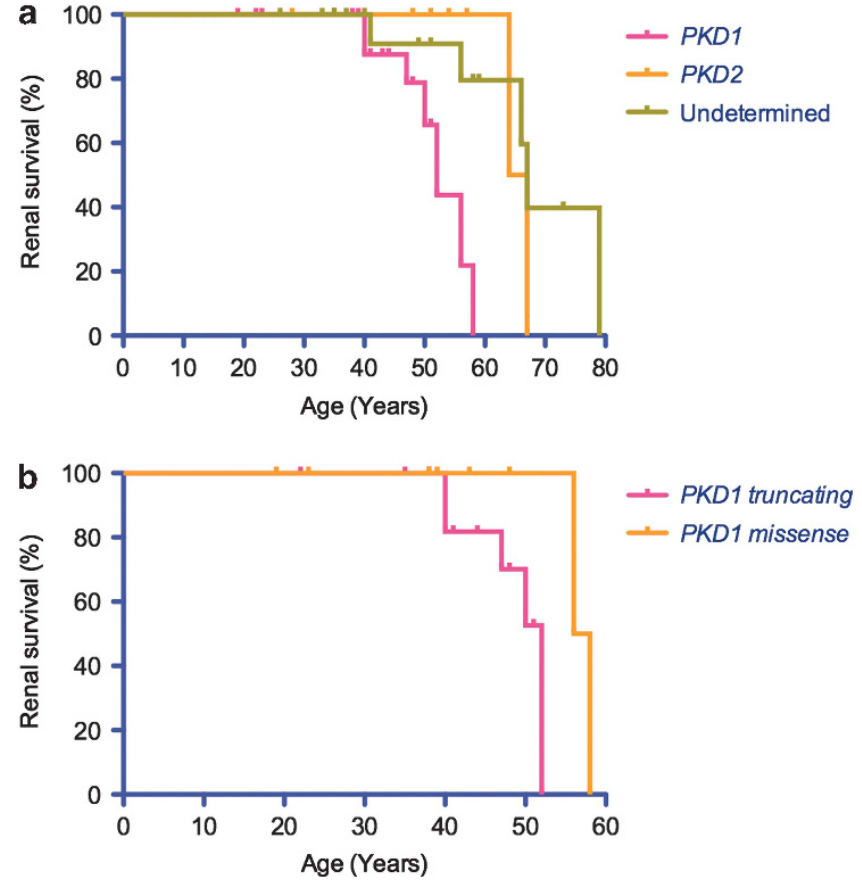

Figure 1 Kaplan-Meier survival curves of the age at onset of ESRD according to $P K D$ genotypes. (a) Comparisons between patients with $P K D 1$ $(n=23)$ and PKD2 mutations $(n=7)$ and patients with an undetermined genotype ( $n=16) \quad(P=0.014$, log-rank test). (b) Comparisons between patients with truncating mutations $(n=14)$ and missense mutations $(n=9)$ in $P K D 1$ in the study cohort $(P=0.061$, log-rank test). Tick marks denote censored patients.

Table 1 Comparison of clinical characteristics between 46 ADPKD patients according to genotypes

\begin{tabular}{|c|c|c|c|c|c|}
\hline & \multirow[b]{2}{*}{ All patients } & \multicolumn{3}{|c|}{ Genotype } & \multirow[b]{2}{*}{ P-value } \\
\hline & & PKD1 $(\mathrm{n}=23)$ & PKD2 $(\mathrm{n}=7)$ & Undetermined $(\mathrm{n}=16)$ & \\
\hline Gender, M/F & $24 / 22$ & $14 / 9$ & $2 / 5$ & $9 / 7$ & 0.32 \\
\hline Age (years) & $47.5 \pm 13.0$ & $43.4 \pm 10.81$ & $52.9 \pm 13.0$ & $51.1 \pm 14.8$ & 0.09 \\
\hline eGFR ( $\mathrm{ml}$ per min per $1.73 \mathrm{~m}^{2}$ ) & $60.2 \pm 45.1$ & $54.8 \pm 41.5$ & $58.6 \pm 39.4$ & $68.61 \pm 53.2$ & 0.65 \\
\hline Positive family $\mathrm{Hx}$ & $35(76 \%)$ & $18(78 \%)$ & $6(86 \%)$ & $11(69 \%)$ & 0.64 \\
\hline Family $\mathrm{Hx}$ of $\mathrm{HD} \leqslant 55 \mathrm{y} / \mathrm{o}$ & $11(24 \%)$ & $9(39 \%)$ & $0(0 \%)$ & $2(13 \%)$ & $0.04^{*}$ \\
\hline Family $\mathrm{Hx}$ of $\mathrm{HD}>65 \mathrm{y} / 0$ & $8(17 \%)$ & $1(4 \%)$ & $3(43 \%)$ & $4(25 \%)$ & $0.04 *$ \\
\hline Diagnosis < 30 y/o & $7(15 \%)$ & $5(22 \%)$ & $1(14 \%)$ & $1(6.25 \%)$ & 0.41 \\
\hline Hypertension & $31(67 \%)$ & $17(74 \%)$ & $5(71 \%)$ & $9(56 \%)$ & 0.50 \\
\hline Gross hematuria & $13(28 \%)$ & $8(35 \%)$ & $1(14 \%)$ & $4(25 \%)$ & 0.54 \\
\hline Urinary tract infection & $17(37 \%)$ & $10(43 \%)$ & $3(43 \%)$ & $4(25 \%)$ & 0.47 \\
\hline Urolithiasis & $19(41 \%)$ & $9(39 \%)$ & $3(43 \%)$ & $7(44 \%)$ & 0.96 \\
\hline Subarachnoid hemorrhage & $1(2 \%)$ & $1(5 \%)$ & $0(0 \%)$ & $0(0 \%)$ & 0.60 \\
\hline Mitral valve prolapse & $1(2 \%)$ & $1(5 \%)$ & $0(0 \%)$ & $0(0 \%)$ & 0.60 \\
\hline Extrarenal cysts (liver, spleen, pancreas) & $29(63 \%)$ & $16(70 \%)$ & $5(71 \%)$ & $8(50 \%)$ & 0.41 \\
\hline Mean kidney length $(\mathrm{cm})$ & $14.8 \pm 2.3$ & $14.9 \pm 2.4$ & $14.7 \pm 1.9$ & $14.8 \pm 2.3$ & 0.99 \\
\hline ACEI/ARB & $28(61 \%)$ & $15(65 \%)$ & $4(57 \%)$ & $9(56 \%)$ & 0.37 \\
\hline
\end{tabular}

Abbreviations: ACEI, angiotensin-converting enzyme inhibitor; ADPKD, autosomal dominant polycystic kidney disease; ARB, angiotensin II receptor blocker; eGFR, estimated glomerular filtration rate; HD, hemodialysis; $\mathrm{Hx}$, history; M, male; F, female.

Data are mean \pm s.d. or number (percentage). ${ }^{*} P<0.05$. 
Table 2 Definite pathogenic mutations in PKD1 found in the study

\begin{tabular}{|c|c|c|c|c|c|c|}
\hline Exon/intron & cDNA change & Protein change & Variant effect & Family no. (Patient no.) & Proband ID & Previous description \\
\hline 5 & c. $1198 \mathrm{C}>\mathrm{T}$ & p.Arg400X & Truncation & 1 & CG9 & $\mathrm{PKDB}^{48}$ \\
\hline 15 & c.3375_3376ins16 & p.Ser1125fs $15 X$ & Frameshift & 1 & CG25 & Novel \\
\hline 15 & c. $4447 \mathrm{C}>\mathrm{T}$ & p.Gln1483X & Truncation & 1 & CG78 & $\mathrm{PKDB}^{39}$ \\
\hline 15 & c.5014_5015delAG & p.Arg1672fs97X & Frameshift & 1 & CG24 & $\mathrm{PKDB}^{25}$ \\
\hline 17 & c. $7204 \mathrm{C}>\mathrm{T}$ & p.Arg2402X & Truncation & 1 & CG67 & $\mathrm{PKDB}^{49}$ \\
\hline 22 & c. $8161+1 G>C$ & & Splicing & 1 & CG13 & Novel \\
\hline 23 & c. $8428 \mathrm{G}>\mathrm{T}$ & p.Glu2810X & Truncation & 1 & CG44 & $\mathrm{PKDB}^{19}$ \\
\hline 23 & c. $8566 \mathrm{C}>\mathrm{T}$ & p.GIn2856X & Truncation & 1 & CG8 & Novel \\
\hline 33 & c.10281ins_C & p.Pro3426fs5X & Frameshift & 1 & CG40 & Novel \\
\hline 35 & c.10528_10529deIAC & p.Thr3509fs136X & Frameshift & 1 & CG29 & Novel \\
\hline 43 & c.11831delT & p.Leu3943fs40X & Frameshift & 1 & CG5 & Novel \\
\hline 45 & c. $12391 \mathrm{G}>\mathrm{T}$ & p.Glu4131X & Truncation & $1(2)$ & CG7 & Novel \\
\hline
\end{tabular}

Abbreviations: ID, identification; PKDB, Polycystic Kidney Mutation Database.

Large duplication is not included in the table.

Table 3 Five PKD2 mutations found in the study

\begin{tabular}{|c|c|c|c|c|c|c|}
\hline Exon & cDNA change & Protein change & Variant effect & Family no. (Patient no.) & Proband ID & Previous description \\
\hline 2 & c. $681 C>A$ & p.Tyr227X & Truncation & 1 & CG62 & $\mathrm{PKDB}^{36}$ \\
\hline 9 & c. $1960 \mathrm{C}>\mathrm{T}$ & p.Arg654X & Truncation & $1(2)$ & CG22 & $\mathrm{PKDB}^{24}$ \\
\hline 13 & c. $2407 \mathrm{C}>\mathrm{T}$ & p.Arg803X & Truncation & $3(9)$ & CG2,CG32,CG73 & $\mathrm{PKDB}^{36,37}$ \\
\hline 5 & c. $1166 \mathrm{C}>\mathrm{T}$ & p.Ala389Val & Substitution & 1 & CG42 & Novel $^{\mathrm{a}}$ \\
\hline 5 & c. $1174 \mathrm{~T}>\mathrm{G}$ & p.Tyr392Asp & Substitution & 1 & CG10 & Novel $^{\mathrm{a}}$ \\
\hline
\end{tabular}

Abbreviations: ID, identification; PKDB, Polycystic Kidney Mutation Database.

apredicted to be likely pathogenic by PolyPhen.

Table 4 Likely pathogenic mutations in PKD1 found in this study and prediction of their pathogenicity

\begin{tabular}{|c|c|c|c|c|c|c|c|c|c|c|c|c|c|c|}
\hline \multirow[b]{2}{*}{ Exon/intron } & \multirow[b]{2}{*}{ cDNA change } & \multirow[b]{2}{*}{$\begin{array}{l}\text { Protein } \\
\text { change }\end{array}$} & \multicolumn{2}{|c|}{ SIFTa } & \multicolumn{2}{|c|}{ SIFTD } & \multicolumn{2}{|c|}{ PolyPhen ${ }^{a}$} & \multicolumn{2}{|c|}{ AlignGVGD } & \multirow[b]{2}{*}{$\begin{array}{l}\text { Family no. } \\
\text { (Patient no.) }\end{array}$} & \multirow[b]{2}{*}{$\begin{array}{l}\text { Proband } \\
\text { ID }\end{array}$} & \multirow[b]{2}{*}{$\begin{array}{l}\text { Frequency } \\
\text { control }\end{array}$} & \multirow[b]{2}{*}{$\begin{array}{l}\text { Previous } \\
\text { description }\end{array}$} \\
\hline & & & $M G$ & VS & $M G$ & VS & $M G$ & VS & $M G$ & VS & & & & \\
\hline 5 & c. $1142 \mathrm{G}>\mathrm{T}$ & p.Gly381Val & $\mathrm{B}$ & 0 & $B$ & 0 & $B$ & 2.40 & $\mathrm{~N}$ & $\mathrm{CO}$ & $1^{\mathrm{c}}$ & CG44 & $0 / 50$ & dbSNP ID: rs2519261 \\
\hline 5 & c. $1211 \mathrm{C}>\mathrm{T}$ & p.Pro404Leu & B & 0 & $\mathrm{~N}$ & 0.11 & $B$ & 2.72 & $\mathrm{~N}$ & $\mathrm{CO}$ & $1(2)$ & CG3 & $0 / 50$ & Novel \\
\hline 5 & c. $1261 \mathrm{C}>\mathrm{T}$ & p.Arg421Cyc & C & 0.01 & C & 0.01 & $B$ & 2.22 & $\mathrm{~N}$ & $\mathrm{CO}$ & 1 & CG53 & $0 / 50$ & Novel \\
\hline 7 & c. $1543 G>A$ & p.Gly515Arg & C & 0.03 & C & 0.02 & B & 2.17 & $\mathrm{~N}$ & $\mathrm{CO}$ & $1^{\mathrm{c}}$ & CG44 & $0 / 50$ & PKDB \\
\hline 15 & c. $6341 A>G$ & p.Tyr2114Сyc & $\mathrm{B}$ & 0 & $B$ & 0 & B & 2.76 & B & C65 & 1 & CG1 & $0 / 50$ & Novel \\
\hline 17 & c. $7172 \mathrm{G}>\mathrm{A}$ & p.Gly2391Asp & $\mathrm{N}$ & 0.66 & B & 0.00 & B & 2.05 & B & C65 & $1(3)$ & CG52 & $0 / 87$ & PKDB $^{39}$ \\
\hline 18 & c. $7430 \mathrm{G}>\mathrm{A}$ & p.Arg2477His & B & 0 & I & 0.05 & C & 1.92 & $\mathrm{~N}$ & CO & $1(2)^{d}$ & CG30 & $7 / 131$ & $\mathrm{PKDB}^{34}$ \\
\hline 23 & c. $8291 \mathrm{~T}>\mathrm{C}$ & p.Met2764Trp & B & 0 & $\mathrm{~N}$ & 0.26 & $B$ & 2.72 & $\mathrm{~N}$ & $\mathrm{CO}$ & $1^{\mathrm{c}}$ & CG44 & $0 / 47$ & Novel \\
\hline 23 & c. $8447 \mathrm{~T}>\mathrm{C}$ & p.Leu2816Pro & C & 0.01 & $\mathrm{~N}$ & 0.11 & $B$ & 2.04 & C & C35 & $1^{\mathrm{c}}$ & CG44 & $0 / 47$ & PKDB $^{50}$ \\
\hline 25 & c. $9157 G>A$ & p.Ala3053Thr & $\mathrm{N}$ & 0.18 & B & 0 & C & 1.54 & B & C55 & $2(2)$ & CG4, CG41 & $0 / 50$ & Novel \\
\hline 28 & c. $9583 \mathrm{~T}>\mathrm{C}$ & p.Trp3195Arg & B & 0 & B & 0 & B & 3.90 & B & C65 & 1 & CG35 & $0 / 50$ & Novel \\
\hline 30 & c. $10043 \mathrm{G}>\mathrm{A}$ & p.Arg3348Gln & I & 0.06 & $B$ & 0.00 & C & 1.75 & C & C35 & $1(2)$ & CG22 & $0 / 59 ; 9 / 11826^{\mathrm{e}}$ & dbSNP ID: rs146494724 51 \\
\hline 31 & c. $10102 \mathrm{G}>\mathrm{A}$ & p.Asp3368Asn & I & 0.05 & C & 0.03 & C & 1.61 & $\mathrm{~N}$ & $\mathrm{co}$ & $2(3)$ & CG7, CG22 & NA & $\mathrm{PKDB}^{30}$ \\
\hline 33 & c. $10315 \mathrm{C}>\mathrm{T}$ & p.Arg3439Trp & $\mathrm{C}$ & 0.01 & $B$ & 0.00 & B & 2.51 & 1 & $\mathrm{C} 25$ & $1(2)^{d}$ & CG30 & $0 / 50 ; 1 / 12972^{\mathrm{e}}$ & ESP ID: TMP_ESP_16_2147410 \\
\hline 38 & c.11157-100_98del3 & Aberrant splicing & & Predic & ted to & 0 introc & duce & new sp & lice sit & & 1 & CG37 & NA & Novel \\
\hline 39 & c. $11257 \mathrm{C}>\mathrm{T}$ & p.Arg3753Trp & 1 & 0.09 & $\mathrm{C}$ & 0.04 & B & 2.00 & $\mathrm{~N}$ & $\mathrm{CO}$ & 1 & CG18 & NA & $\mathrm{PKDB}^{25}$ \\
\hline
\end{tabular}

Abbreviations: dbSNP, database for single-nucleotide polymorphism; ESP, Exome Sequencing Project; ID, identification; MG, mutation group (B: highly likely; C: likely; I: indeterminate; N: neutral); NA, not analyzed; PKDB, Polycystic Kidney Mutation Database; VS, variant score.

a Default alignment (human, mouse, rat, chicken, xenopus, fugu).

bPolycystin-1 orthologue alignment (human, mouse, rat, chicken, xenopus, fugu, dog, opossum, tetraodon nigroviridis)

'Found with p.E2810X in CG44.

dOccurred simultaneously.

eAllele frequencies in ESP.

\section{Large duplication mutation}

To identify large deletion or duplication mutation that cannot be detected by direct sequencing, we then performed a copy number analysis using MLPA on all patients in this cohort. There were no multiple exon deletions found. Duplication of PKD1 exon 1 (relative peak ratio 1.66) was identified in a 47-year-old woman, and a similar result was found in her son (relative peak ratio: 1.58) (Figure 2a). Repeated MLPA gave similar results. These results were further 
validated using real-time Q-PCR (Figure $2 \mathrm{~b}$ ). The pedigree of this family and clinical data are shown in Figure 2c.

\section{Hypomorphic alleles}

In one family, the concurrence of PKD1 p.Arg2477His and p.Arg3439Trp variants segregated with the presence of polycystic kidney disease in two sisters (Figure 3a). To our surprise, their parents and two other siblings, each of them carries PKD1 p.Arg3439Trp or p.Arg2477His, were negative for renal cysts. These results suggested that p.Arg3439Trp and p.Arg2477His could be hypomorphic alleles, as both were incompletely penetrant. PKD1 p.Arg2477His has been previously reported in a Han Chinese family as a pathogenic mutation, ${ }^{34}$ but whether or not it coexisted with other missense mutations is not known. PKD1 p.Arg3439Trp is predicted to be highly likely pathogenic by computer programs, and its allele frequency in the Exome Sequencing Project was 1/ 12972 (Table 4). Amino-acid multi-alignments demonstrate that the positions of mutations are highly conserved across species in p.Arg3439Trp and partially conserved in p.Arg2477His (Figure 3b).

\section{Undetermined genotype}

There were 16 patients who had no PKD variants found or the identified sequence variants were interpreted as non-pathogenic. a
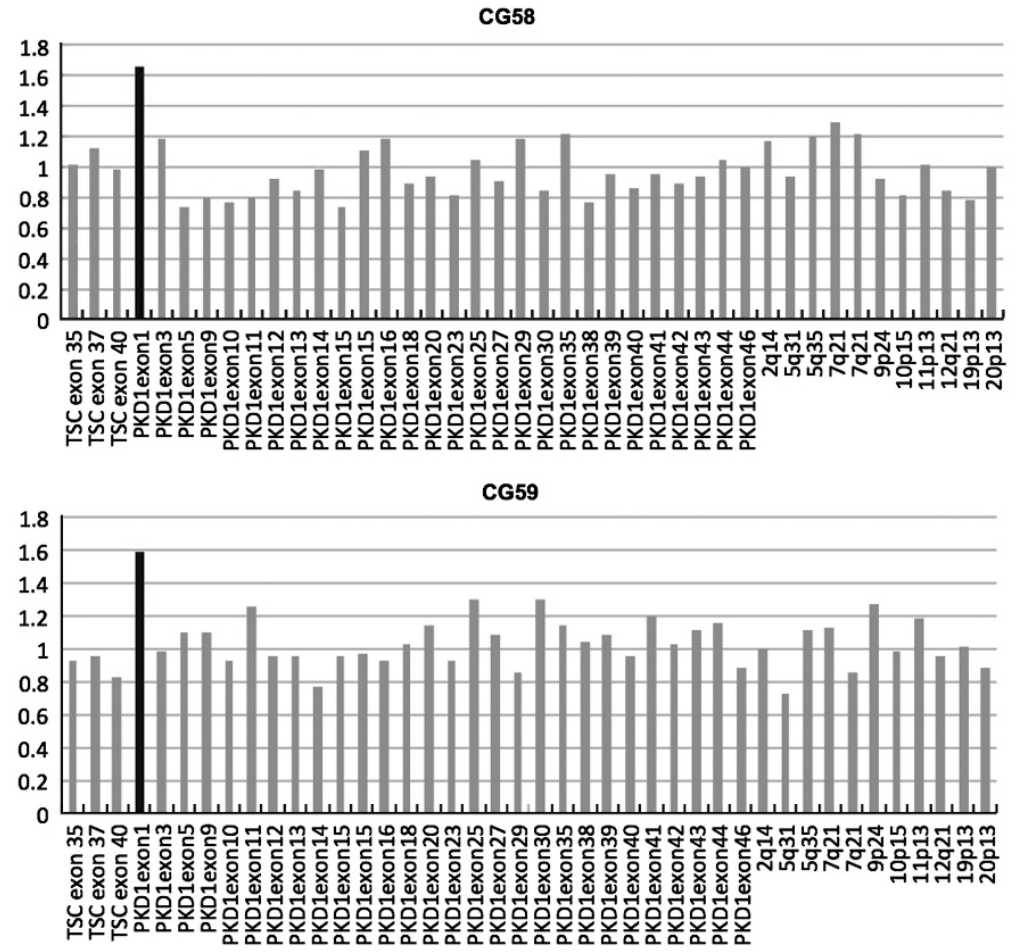

b

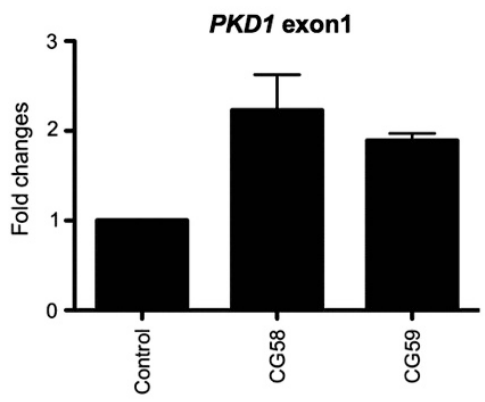

c

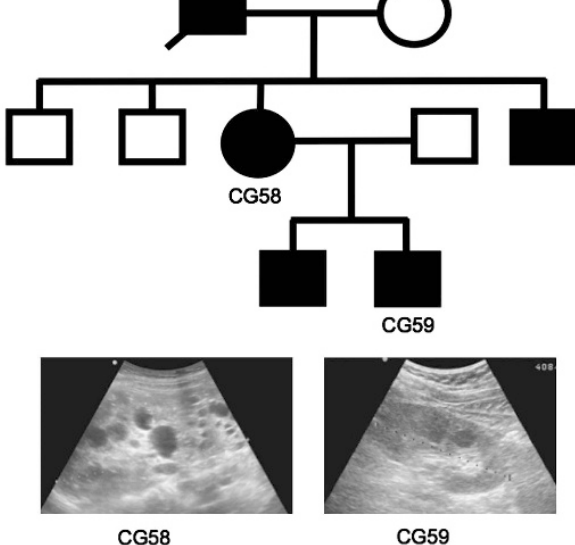

Figure 2 MLPA analysis showing duplication of PKD1 exon 1 in a Taiwanese family of ADPKD. (a) PKD1 and reference probes are listed on the $x$ axis. The relative peak area of the amplification product (peak ratio) is depicted on the $y$ axis. Note that the peak ratios of PKD1 exon 1 probe (black bars) are increased in patient CG 58 (1.63) and patient CG59 (1.58). (b) Pedigree and ultrasonography of the family. CG58 (mother) had bilateral enlarged kidneys with multiple cysts at the age of 47 years (eGFR: $77 \mathrm{ml}$ per min per $1.73 \mathrm{~m}^{2}$ ). Her father required dialysis at the age of 64 years and died after 7 years. CG59 had a few cysts at the age of 18 years with normal renal function. Other family members were unavailable for genetic testing. (c) Real-time Q-PCR using Custom Taqman Copy Number Assays with primers against the binding site of the MLPA exon 1 probe. Results shown are means \pm s.d. from two independent experiments done in triplicate. 
a

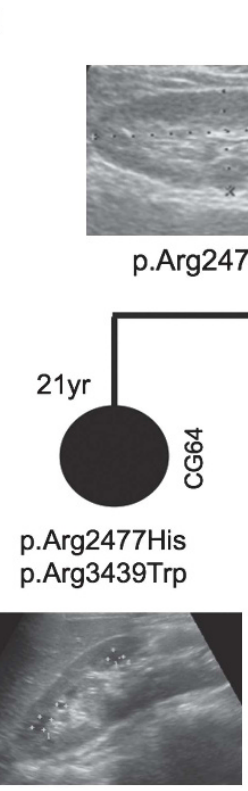

$57 \mathrm{yr}$
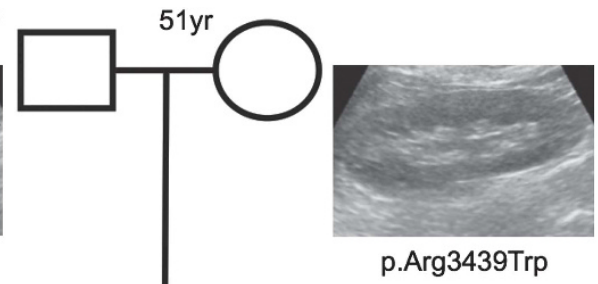

p.Arg3439Trp

b

\begin{tabular}{|c|c|c|c|c|c|c|c|c|}
\hline & \multicolumn{5}{|c|}{ p.Arg2477His } & \multicolumn{3}{|c|}{ p.Arg3439Trp } \\
\hline Human & \multicolumn{5}{|c|}{$\frac{\mathrm{H}}{\text { IRLSPNRPPLGGSCRLFPLG }}$} & \multicolumn{3}{|c|}{ SNLRQLARGOAGHGLGPE } \\
\hline Mouse & \multicolumn{5}{|c|}{ IRLSPNRPPLGGSCRLFPLD } & \multicolumn{3}{|c|}{ STLQRLTQGRPGCMLGSE } \\
\hline Rat & \multicolumn{5}{|c|}{ IRLLPNRPPLGGSCRLFPLE } & \multicolumn{3}{|c|}{ STLQRLAQGRPGCMLGSE } \\
\hline Opos sum & \multicolumn{5}{|c|}{ IDLFPNRPPIGGSCRLFPHE } & \multicolumn{3}{|c|}{ NTLQRLVRARAGNPPGLE } \\
\hline Dog & \multicolumn{5}{|c|}{ IRLSANRPPRGGSCRLFPLD } & \multicolumn{3}{|c|}{ STLQRLARGRTGHTLGPE } \\
\hline Chicken & \multicolumn{5}{|c|}{ IDLLPNQPPIDGSCQLSPKG } & \multicolumn{3}{|c|}{ NTIQKLKRGRASRHLGLE } \\
\hline Xenopus & \multicolumn{5}{|c|}{ IDLLPNSPPSGGSCTISPNG } & \multicolumn{3}{|c|}{ NTIQKLERRRTIRHLGLD } \\
\hline Fugu & \multicolumn{5}{|c|}{ ITLHHNMPPDGGECHLRRGG } & \multirow{2}{*}{\multicolumn{3}{|c|}{ AGLPRLKRGQGSRHLGVD }} \\
\hline Tet raodon & \multicolumn{5}{|c|}{ ITLNHNMPPDGGECHLRREG } & & & PGLARLKRGQGSRHLGLD \\
\hline Variant & \multicolumn{2}{|c|}{ SIFT' $^{2}$} & \multicolumn{2}{|c|}{$\mathrm{SIFT}^{\mathrm{b}}$} & \multicolumn{2}{|c|}{ PolyPhen ${ }^{\text {a }}$} & \multicolumn{2}{|c|}{ AlignGVGD } \\
\hline & MG & vs & $M C$ & vs & $\mathrm{MG}$ & vs & MG & vs \\
\hline p.Arg2477 & $B$ & 0 & & 0.05 & C & 1.92 & $\mathrm{~N}$ & CO \\
\hline & & & & & B & & & \\
\hline
\end{tabular}

Figure 3 Two PKD1 missense mutations (p.Arg2477His and p.Arg3439Trp) segregated with ADPKD in a Taiwanese family. (a) The pedigree and renal ultrasound data of the family. These mutations segregated in two affected daughters with multiple renal cysts. The parents and two other siblings had normal renal function, and the findings of renal ultrasound were normal. The proband (CG 30) presented with recurrent urinary tract infection at the age of 19 years and bilateral renal cysts and ovarian cysts were found (eGFR: $89 \mathrm{ml}$ per min per $1.73 \mathrm{~m}^{2}$ ). Her sister (CG 63) presented with bilateral renal cysts at the age of 21 years (eGFR: $109 \mathrm{ml}$ per min per $1.73 \mathrm{~m}^{2}$ ) and had a history of acute pyelonephritis. (b) Multiple sequence alignments for the PKD1 changes p.Arg2477His and p.Arg3439Trp. The results of pathogenicity prediction using web-based computer programs (SIFT, Polyphen and AlignGVGD) are

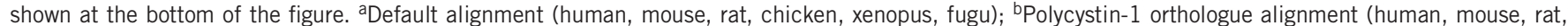
chicken, xenopus, fugu, dog, opossum, tetraodon nigroviridis); MG, mutation group (B: highly likely; C: likely; I, indeterminate: N, neutral); VS, variant score.

These patients tended to have a slower decline of renal function than the patients with PKD1 mutations (Figure 1a). Other clinical characteristics were not significantly different in patients with an undetermined genotype compared with others (Table 1).

\section{DISCUSSION}

We performed a comprehensive clinical and mutational analysis in a cohort of ADPKD patients. This is the first study to describe the characteristics of $P K D 1$ and PKD2 mutations in Taiwan. Worldwide, there have been 1923 sequence variants for $P K D 1$ and 241 for $P K D 2$ as described in the PKDB. Among these variants, 929 of $P K D 1$ and 167 of $P K D 2$ are pathogenic mutations. In the current study, 50\% (17/34) of mutations are novel and 50\% (17/34) are recurrent mutations reported in the literature. The 17 novel mutations we found in the Taiwanese population would expand the spectrum of $P K D$ mutations and contribute to the global references for genetic counseling of ADPKD patients.

Among the 34 mutations we identified, only three mutations were present in more than one unrelated patient. This is consistent with the findings that no single mutation accounts for $>2 \%$ of unrelated ADPKD patients in previous studies. ${ }^{2,35}$ Therefore, ADPKD patients who seek for the genetic diagnosis need to undergo a complete mutation analysis of PKD1 and PKD2 unless the causative mutations have been identified in other family members. Nevertheless, a recurrent mutation PKD2 p.R803X was found in three families in Taiwan and five families in other populations. ${ }^{30,36-39}$ This frequent mutation could be related to its location in the $\mathrm{CpG}$ dinucleotide site that is susceptible to 
mutations, or it could be a founder mutation that derived from the common ancestor. ${ }^{35}$

The frequency of large rearrangements reported in a large ADPKD cohort $(n=202)$ was $4 \% .{ }^{17}$ In this study, we identified duplication of PKD1 exon 1 by MLPA and Q-PCR in 1 out of 46 cases (2\%). A previous study has reported duplication of PKD1 IVS17 and exon 18 in one patient. ${ }^{17}$ Overall, deletion and duplication mutations are rare in ADPKD patients, and MLPA can be reserved for those mutationnegative patients after direct PCR sequencing.

Knowing whether a family carries mutations in PKD1 or PKD2 may have prognostic value, because PKD2 patients typically develop ESRD two decades later than PKD1 patients. ${ }^{26}$ Furthermore, the mutation type and location in PKD1 have been shown to influence renal survival in previous studies. ${ }^{15}$ The position of the mutation in $P K D 1$ has been shown to predict the occurrence of intracranial aneurysms. ${ }^{40}$ In a recent report from 519 pedigrees in the Genkyst cohort, carriers of a PKD1-truncating mutation have a significantly earlier age at onset of ESRD than patients with a non-truncating mutation (55 years vs 67 years). ${ }^{14}$ Our data support the notion that a more severe phenotype can be expected in PKD1 patients, especially in those with a PKD1truncating mutation. Therefore, genetic testing for ADPKD patients would be helpful to clarify the disease status and provide prognostic information. ${ }^{2}$ Robinson et al. ${ }^{26}$ proposed that direct PKD2 mutation testing can be offered to identify the subgroup less likely to progress to ESRD. The prognostic information could be important for guiding the choice of potential treatments for ADPKD that might have side effects after long-term use. ${ }^{28,41}$

Hypomorphic PKD1 alleles are increasingly recognized in recent genotype-phenotype studies. ${ }^{20-22}$ These missense variants have reduced PC-1 function and cause mild disease when present alone or severe disease when they occur simultaneously. In our cohort, we found two likely hypomorphic alleles, PKD1 p.Arg2477His and p.Arg3439Trp, which segregated with the disease in one family. To date, nine PKD1 hypomorphic alleles have been described in different populations as collated in PKDB. ${ }^{21,22}$ For example, a compound heterozygote for the $P K D 1$ variants p.Arg3105Trp and p.Arg2765Cys caused multiple cysts in childhood, whereas another family member heterozygous for p.Arg2765Cys developed only one cyst. ${ }^{20}$ Patients heterozygous for PKD1 p.Arg3277Cys had only few cysts, but those homozygous for p.Arg3277Cys developed multiple renal cysts and ESRD. ${ }^{20}$ PKD1 p.Arg2220Trp and p.Arg3277Cyc in trans co-segregated with early-onset ADPKD, whereas no cysts were found in the heterozygous parents. ${ }^{21}$ These findings support the concept of a critical cellular threshold of PCs in the pathogenesis of ADPKD. ${ }^{22,42}$ A valid functional study for these missense variants is still lacking. ${ }^{22,43}$

It is worth noting that a careful history taking for the age of ESRD in affected family members may provide a simple means of predicting the mutated gene in clinical practice. ${ }^{13}$ Our results support the use of family history information as an aid in risk stratification. For those whose relatives have developed ESRD before the age of 50 years, the family history is highly suggestive of carrying a PKD1 mutation. ${ }^{13,26}$ For those whose affected members developed ESRD aged $>70$ years or continued to have sufficient renal function, the presence of a PKD2 mutation is highly likely. ${ }^{13}$

In previous studies, disease-causing mutations were identified in approximately $64-90 \%$ of ADPKD patients by direct PCR sequencing. ${ }^{35,39}$ Another study using denaturing high-performance liquid chromatography found mutations in only $52 \%$ of ADPKD patients. ${ }^{44}$ The possible causes of the relative low mutation detection rate in our study may include cohort size, methods of mutational analysis, sequencing technique, the presence of large deletion/duplication, deep intron sequence, mosaicism or mutations in other genes. In addition, some missense mutations that were incompletely penetrant could be interpreted as sequence variants. Furthermore, patients without a family history of ADPKD were included in our study. It seems that definite pathogenic mutations are found more frequently in patients with a positive family history of ADPKD than in those without. $^{2,35}$

Several potential limitations of this study merit consideration. First, we only screened for PKD1 and PKD2 mutations and therefore cannot exclude the existence of other mutations such as HNF1 $\beta$, PRKCSH, SEC63 or PKHD1 that may contribute to the cystic phenotype. $^{2}$ Second, the expensiveness of exon by exon DNA sequencing precludes us from recruiting more cases. Relatively small sample size may limit the ability to detect a small phenotypic difference between genotypes. Nevertheless, we were still able to detect phenotype differences in our study cohort. Finally, the likely pathogenic mutations identified in our study need to be confirmed in future studies involving more ADPKD families.

As potential therapies for ADPKD are being developed, molecular testing will likely become increasingly important. ${ }^{2,28}$ Another important indication is to determine the disease status of potential living related donors with equivocal imaging findings. ${ }^{19,45} \mathrm{~A}$ recent study has shown great promise of next-generation sequencing for genetic PKD diagnostics, because the time and effort needed to detect pathogenic mutations are markedly reduced. ${ }^{46}$ Therefore, more efficient and low-cost genetic testing can be expected in the next decade, with the advance of DNA sequencing technique. ${ }^{35,46}$ In addition to genetic testing, a simple family history enquiry for the age of hemodialysis may yield prognostic information by estimating PKD1 (early dialysis) and PKD2 (late dialysis) genotypes. ${ }^{13}$ Many clinical trials of different mechanism-based drug treatment for ADPKD are currently undergoing. ${ }^{47}$ It would be interesting to know whether patients with different $P K D$ mutations may respond differently to these novel treatments.

\section{CONCLUSIONS}

We presented the first complete clinical and mutational analysis of PKD1/PKD2 in Taiwanese patients with ADPKD. We identified 17 novel mutations of $P K D 1$ and $P K D 2$ and demonstrated the presence of PKD1 hypomorphic alleles. Patients carrying PKD1 mutations, especially those with truncating mutations, could have a more rapidly progressive disease than others. These results might have implications for diagnosis and risk stratification in patients with ADPKD.

\section{CONFLICT OF INTEREST}

The authors declare no conflict of interest.

\section{ACKNOWLEDGEMENTS}

We thank Dr Sandro Rossetti for helpful discussion and technical advice. We appreciate Professor Chin-Song Lu for providing control DNA samples. We thank Chiung-Tseng Huang for her excellent technical assistance and Dr YenYu Liau for data collection. This study was supported by grants from the Chang Gung Memorial Hospital (CMRPG 370941/370942 and CMRPG 391651). 
1 Torres, V. E., Bankir, L. \& Grantham, J. J. A case for water in the treatment of polycystic kidney disease. Clin. J. Am. Soc. Nephrol. 4, 1140-1150 (2009).

2 Harris, P. C. \& Rossetti, S. Molecular diagnostics for autosomal dominant polycystic kidney disease. Nat. Rev. Nephrol. 6, 197-206 (2010).

3 Chang, M. Y. \& Ong, A. C. Autosomal dominant polycystic kidney disease: recent advances in pathogenesis and treatment. Nephron Physiol. 108, p1-p7 (2008).

4 Newby, L. J., Streets, A. J., Zhao, Y., Harris, P. C., Ward, C. J. \& Ong, A. C. Identification, characterization, and localization of a novel kidney polycystin-1-polycystin-2 complex. J. Biol. Chem. 277, 20763-20773 (2002).

5 Bertuccio, C. A., Chapin, H. C., Cai, Y., Mistry, K., Chauvet, V., Somlo, S. et al. Polycystin-1 C-terminal cleavage is modulated by polycystin-2 expression. J. Biol. Chem. 284, 21011-21026 (2009).

6 Parnell, S. C., Puri, S., Wallace, D. P. \& Calvet, J. P. Protein phosphatase-1alpha interacts with and dephosphorylates polycystin-1. PLoS One 7, e36798 (2012).

7 Nauli, S. M., Alenghat, F. J., Luo, Y., Williams, E., Vassilev, P., Li, X. et al. Polycystins 1 and 2 mediate mechanosensation in the primary cilium of kidney cells. Nat. Genet. 33, 129-137 (2003).

8 Luyten, A., Su, X., Gondela, S., Chen, Y., Rompani, S., Takakura, A. et al. Aberrant regulation of planar cell polarity in polycystic kidney disease. J. Am. Soc. Nephrol. 21, 1521-1532 (2010).

9 Streets, A. J., Wagner, B. E., Harris, P. C., Ward, C. J. \& Ong, A. C. Homophilic and heterophilic polycystin 1 interactions regulate $\mathrm{E}$-cadherin recruitment and junction assembly in MDCK cells. J. Cell. Sci. 122, 1410-1417 (2009).

10 Drummond, I. A. Polycystins, focal adhesions and extracellular matrix interactions. Biochim. Biophys. Acta 1812, 1322-1326 (2011).

11 Johnson, A. M. \& Gabow, P. A. Identification of patients with autosomal dominant polycystic kidney disease at highest risk for end-stage renal disease. J. Am. Soc. Nephrol. 8, 1560-1567 (1997).

12 Harris, P. C., Bae, K. T., Rossetti, S., Torres, V. E., Grantham, J. J., Chapman, A. B. et al. Cyst number but not the rate of cystic growth is associated with the mutated gene in autosomal dominant polycystic kidney disease. J. Am. Soc. Nephrol. 17, 30133019 (2006).

13 Barua, M., Cil, O., Paterson, A. D., Wang, K., He, N., Dicks, E. et al. Family history of renal disease severity predicts the mutated gene in ADPKD. J. Am. Soc. Nephrol. 20 , 1833-1838 (2009).

14 Cornec-Le Gall, E., Audrezet, M. P., Chen, J. M., Hourmant, M., Morin, M. P., Perrichot, R. et al. Type of PKD1 mutation influences renal outcome in ADPKD. J. Am. Soc. Nephrol. 24, 1006-1013 (2013).

15 Rossetti, S., Burton, S., Strmecki, L., Pond, G. R., San Millan, J. L., Zerres, K. et al. The position of the polycystic kidney disease 1 (PKD1) gene mutation correlates with the severity of renal disease. J. Am. Soc. Nephrol. 13, 1230-1237 (2002).

16 Pei, Y., Obaji, J., Dupuis, A., Paterson, A. D., Magistroni, R., Dicks, E. et al. Unified criteria for ultrasonographic diagnosis of ADPKD. J. Am. Soc. Nephrol. 20, 205-212 (2009).

17 Consugar, M. B., Wong, W. C., Lundquist, P. A., Rossetti, S., Kubly, V. J., Walker, D. L. et al. Characterization of large rearrangements in autosomal dominant polycystic kidney disease and the PKD1/TSC2 contiguous gene syndrome. Kidney Int. 74, 1468-1479 (2008).

18 Kozlowski, P., Bissler, J., Pei, Y. \& Kwiatkowski, D. J. Analysis of PKD1 for genomic deletion by multiplex ligation-dependent probe assay: absence of hot spots. Genomics 91, 203-208 (2008).

19 Garcia-Gonzalez, M. A., Jones, J. G., Allen, S. K., Palatucci, C. M., Batish, S. D., Seltzer, W. K. et al. Evaluating the clinical utility of a molecular genetic test for polycystic kidney disease. Mol. Genet. Metab. 92, 160-167 (2007).

20 Rossetti, S., Kubly, V. J., Consugar, M. B., Hopp, K., Roy, S., Horsley, S. W. et al. Incompletely penetrant PKD1 alleles suggest a role for gene dosage in cyst initiation in polycystic kidney disease. Kidney Int. 75, 848-855 (2009).

21 Vujic, M., Heyer, C. M., Ars, E., Hopp, K., Markoff, A., Orndal, C. et al. Incompletely penetrant PKD1 alleles mimic the renal manifestations of ARPKD. J. Am. Soc. Nephrol. 21, 1097-1102 (2010).

22 Pei, Y., Lan, Z., Wang, K., Garcia-Gonzalez, M., He, N., Dicks, E. et al. A missense mutation in PKD1 attenuates the severity of renal disease. Kidney Int. 81, 412-417 (2012).

23 Torres, V. E. \& Harris, P. C. Autosomal dominant polycystic kidney disease: the last 3 years. Kidney Int. 76, 149-168 (2009).

24 Irazabal, M. V., Huston, J. 3rd, Kubly, V., Rossetti, S., Sundsbak, J. L., Hogan, M. C. et al. Extended follow-up of unruptured intracranial aneurysms detected by presymptomatic screening in patients with autosomal dominant polycystic kidney disease. Clin. J. Am. Soc. Nephrol. 6, 1274-1285 (2011).

25 Rossetti, S., Consugar, M. B., Chapman, A. B., Torres, V. E., Guay-Woodford, L. M., Grantham, J. J. et al. Comprehensive molecular diagnostics in autosomal dominant polycystic kidney disease. J. Am. Soc. Nephrol. 18, 2143-2160 (2007).

26 Robinson, C., Hiemstra, T. F., Spencer, D., Waller, S., Daboo, L., Karet Frankl, F. E. et al. Clinical utility of PKD2 mutation testing in a polycystic kidney disease cohort attending a specialist nephrology out-patient clinic. BMC Nephrol. 13, 79 (2012).
27 Ravine, D., Gibson, R. N., Walker, R. G., Sheffield, L. J., Kincaid-Smith, P. \& Danks, D. M. Evaluation of ultrasonographic diagnostic criteria for autosomal dominant polycystic kidney disease 1. Lancet 343, 824-827 (1994).

28 Torres, V. E., Chapman, A. B., Devuyst, O., Gansevoort, R. T., Grantham, J. J., Higashihara, E. et al. Tolvaptan in patients with autosomal dominant polycystic kidney disease. N. Engl. J. Med. 367, 2407-2418 (2012).

29 Stevens, L.A., Coresh, J., Greene, T. \& Levey, A.S. Assessing kidney function-measured and estimated glomerular filtration rate. N. Engl. J. Med. 354, 2473-2483 (2006).

30 Zhang, S., Mei, C., Zhang, D., Dai, B., Tang, B., Sun, T. et al. Mutation analysis of autosomal dominant polycystic kidney disease genes in Han Chinese. Nephron Exp. Nephrol. 100, e63-e76 (2005).

31 Rossetti, S., Chauveau, D., Walker, D., Saggar-Malik, A., Winearls, C. G., Torres, V. E. et al. A complete mutation screen of the ADPKD genes by DHPLC. Kidney Int. 61, 1588-1599 (2002).

32 Desmet, F.O., Hamroun, D., Lalande, M., Collod-Beroud, G., Claustres, M. \& Beroud, C. Human Splicing Finder: an online bioinformatics tool to predict splicing signals. Nucleic Acids Res. 37, e67 (2009).

33 Losekoot, M., Ruivenkamp, C. A., Tholens, A. P., Grimbergen, J. E., Vijfhuizen, L., Vermeer, S. et al. Neonatal onset autosomal dominant polycystic kidney disease (ADPKD) in a patient homozygous for a PKD2 missense mutation due to uniparental disomy. J. Med. Genet. 49, 37-40 (2012).

34 Zhang, S. Z., Zhang, Y. H., Zhang, D. Y. \& Mei, C. L. Mutation detection of ADPKD PKD1 gene in Hans by denaturing high-performance liquid chromatography. Chin. J. Med. Genet. 23, 283-288 (2006).

35 Audrezet, M. P., Cornec-Le Gall, E., Chen, J. M., Redon, S., Quere, I., Creff, J. et al. Autosomal dominant polycystic kidney disease: comprehensive mutation analysis of PKD1 and PKD2 in 700 unrelated patients. Hum. Mutat. 33, 1239-1250 (2012).

36 Chang, M. Y., Fang, J. T., Huang, C. C., Wu, I. W. \& Wu Chou, Y. H. Mutations of the PKD2 gene in Taiwanese patients with autosomal dominant polycystic kidney disease. Ren. Fail. 27, 95-100 (2005).

37 Deltas, C. C. Mutations of the human polycystic kidney disease 2 (PKD2) gene. Hum. Mutat. 18, 13-24 (2001).

38 Chung, W., Kim, H., Hwang, Y. H., Kim, S. Y., Ko, A. R., Ro, H. et al. PKD2 gene mutation analysis in Korean autosomal dominant polycystic kidney disease patients using two-dimensional gene scanning. Clin. Genet. 70, 502-508 (2006).

39 Hoefele, J., Mayer, K., Scholz, M. \& Klein, H. G. Novel PKD1 and PKD2 mutations in autosomal dominant polycystic kidney disease (ADPKD). Nephrol. Dial. Transplant. 26, 2181-2188 (2011).

40 Rossetti, S., Chauveau, D., Kubly, V., Slezak, J. M., Saggar-Malik, A. K., Pei, Y. et al. Association of mutation position in polycystic kidney disease 1 (PKD1) gene and development of a vascular phenotype. Lancet 361, 2196-2201 (2003).

41 Walz, G., Budde, K., Mannaa, M., Nurnberger, J., Wanner, C., Sommerer, C. et al. Everolimus in patients with autosomal dominant polycystic kidney disease. N. Engl. J. Med. 363, 830-840 (2010).

42 Hopp, K., Ward, C. J., Hommerding, C. J., Nasr, S. H., Tuan, H. F., Gainullin, V. G. et al. Functional polycystin-1 dosage governs autosomal dominant polycystic kidney disease severity. J. Clin. Invest. 122, 4257-4273 (2012).

$43 \mathrm{Ma}$, L., Xu, M., Forman, J. R., Clarke, J. \& Oberhauser, A. F. Naturally occurring mutations alter the stability of polycystin-1 polycystic kidney disease (PKD) domains. J. Biol. Chem. 284, 32942-32949 (2009).

44 Yu, C., Yang, Y., Zou, L., Hu, Z., Li, J., Liu, Y. et al. Identification of novel mutations in Chinese Hans with autosomal dominant polycystic kidney disease. BMC Med. Genet. 12, 164 (2011).

45 Huang, E., Samaniego-Picota, M., McCune, T., Melancon, J. K., Montgomery, R. A., Ugarte, R. et al. DNA testing for live kidney donors at risk for autosomal dominant polycystic kidney disease. Transplantation 87, 133-137 (2009).

46 Rossetti, S., Hopp, K., Sikkink, R. A., Sundsbak, J. L., Lee, Y. K., Kubly, V. et al. Identification of gene mutations in autosomal dominant polycystic kidney disease through targeted resequencing. J. Am. Soc. Nephrol. 23, 915-933 (2012).

47 Chang, M. Y. \& Ong, A. C. Mechanism-based therapeutics for autosomal dominant polycystic kidney disease: recent progress and future prospects. Nephron Clin. Pract 120, c25-c34, discussion c35 (2012)

48 Peters, D. J., Ariyurek, Y., van Dijk, M. \& Breuning, M. H. Mutation detection for exons 2 to 10 of the polycystic kidney disease 1 (PKD1)-gene by DGGE. Eur. J. Hum. Genet. 9, 957-960 (2001).

49 Phakdeekitcharoen, B., Watnick, T. J., Ahn, C., Whang, D. Y., Burkhart, B. \& Germino, G. G. Thirteen novel mutations of the replicated region of PKD1 in an Asian population. Kidney Int. 58, 1400-1412 (2000).

50 Rossetti, S., Strmecki, L., Gamble, V., Burton, S., Sneddon, V., Peral, B. et al. Mutation analysis of the entire PKD1 gene: genetic and diagnostic implications. Am. J. Hum. Genet. 68, 46-63 (2001).

51 Bataille, S., Berland, Y., Fontes, M. \& Burtey, S. High resolution melt analysis for mutation screening in PKD1 and PKD2. BMC Nephrol. 12, 57 (2011). 